\title{
Algorithm to Mine Negative Association Rules
}

\author{
B.Ramasubbareddy ${ }^{1}$, K.Sekar. ${ }^{2}$, Dr.A.Ramamohanreddy ${ }^{3}$ \\ Professor, S.V.College of Engineering, Tirupathi, India ${ }^{1}$ \\ Professor, S.V.College of Engineering, Tirupathi, India ${ }^{2}$ \\ Professor, S.V.U. College of Engineering, S.V.University, Tirupati, India ${ }^{3}$
}

\begin{abstract}
Association rule mining is one of the most popular data mining techniques to find associations among items in a set by mining necessary patterns in a large database. Typical association rules consider only items enumerated in transactions. Such rules are referred to as positive association rules. Negative association rules also consider the same items, but in addition consider negated items (i.e. absent from transactions). Negative association rules are useful in market-basket analysis to identify products that conflict with each other or products that complement each other. They are also very useful for constructing associative classifiers. In this paper, we propose an algorithm that mines positive and negative association rules without adding any additional measure and extra database scans.
\end{abstract}

KEYWORDS: Data Mining, Negative Association Rules, Support, Confidence.

\section{INTRODUCTION}

Association rule mining is a data mining task that discovers associations among items in a transactional database. Association rules have been extensively studied in the literature for their usefulness in many application domains such as recommender systems, diagnosis decisions support, telecommunication, intrusion detection, etc. Efficient discovery of such rules has been a major focus in the data mining research. From the celebrated Apriori algorithm [1] there have been a remarkable number of variants and improvements of association rule mining algorithms [2]. A typical example of association rule mining application is the market basket analysis. In this process, the behavior of the customers is studied with reference to buying different products in a shopping store. The discovery of interesting patterns in this collection of data can lead to important marketing and management strategic decisions. For instance, if a customer buys bread, what are chances that customer buys milk as well? Depending on some measure to represent the said chances of such an association, marketing personnel can develop better planning of the shelf space in the store or can base their discount strategies on such associations/correlations found in the data. All the traditional association rule mining algorithms were developed to find positive associations between items. By positive associations, we refer to associations between items exist in transactions containing the items bought together. What about associations of the type: "customers that buy Coke do not buy Pepsi" or "customers that buy juice do not buy bottled water"? In addition to the positive associations, the negative association can provide

valuable information, in devising marketing strategies. This paper is structured as follows: the next section recalls preliminaries about Association Rules, In Section 3, existing strategies for mining negative Association Rules are reviewed. The proposed algorithm is presented in Section 4 and this is a new Apriori-based algorithm for finding all valid positive and negative association rules. Section 5 contains conclusions and future work.

\section{BASIC CONCEPTS AND TERMINOLOGY}

This section introduces association rules terminology and some related work on negative association rules.

\section{A. Association Rules}

Formally, association rules are defined as follows: Let $I=\left\{i 1, i 2, \ldots i_{n}\right\}$ be a set of items. Let $D$ be a set of transactions, where each transaction $T$ is a set of items such that $T \subseteq I$.

Each transaction is associated with a unique identifier TID. A transaction $T$ is said to contain $X$, a set of items in $I$, if $X \subseteq T$. An association rule is an implication of the form " $X \quad Y$ ", where $X \subseteq I ; Y \subseteq I$, and $X \quad Y=$. The rule $X \quad Y$ has support s in the transaction set $D$ if $s \%$ of the transactions in $D$ contain $X U Y$. In other words, the support of the rule is the probability that $X$ and $Y$ hold together among all the possible presented cases. It is said that the rule $X \quad Y$ holds in the transaction set $D$ with confidence $c$ if $c \%$ of transactions in $D$ that contain $X$ also contain $Y$. In other words, the confidence of

the rule is the conditional probability that the consequent $Y$ is true under the condition of the antecedent $X$. The problem of discovering all association rules from a set of transactions $D$ consists of generating the rules that have a support and confidence greater than given thresholds. These rules are called strong rules, and the framework is known as the support-confidence framework for association rule mining. Definition of Negative Association Rule A negative association rule is an implication of the form $X \quad Y$

(or $X \quad Y$ or $X \quad Y$ ), where $X \subseteq I, Y \subseteq I$ and 


\title{
International Journal of Innovative Research in Science, Engineering and Technology
}

\author{
Vol. 1, Issue 1, November 2012
}

$X \quad Y=$ (Note that although rule in the form of $X \quad Y$ contains negative elements, it is equivalent to a positive association rule in the form of $Y \quad X$. Therefore it is not considered as a negative association rule.) In contrast to positive rules, a negative rule encapsulates relationship between the occurrences of one set of items with the absence of the other set of items. The rule $X \quad Y$ has support s $\%$ in the data sets, if $s \%$ of transactions in $T$ contain itemset $X$ while do not contain itemset $Y$. The support of a negativ association rule, $\operatorname{supp}\left(\begin{array}{ll}X & Y\end{array}\right)$, is the frequency of occurrence of transactions with item set $X$ in the absence of item set $Y$. Let $U$ be the set of transactions that contain all items in $X$. The rule $X \quad Y$ holds in the given data set (database) with confidence $c \%$, if $\mathrm{c} \%$ of transactions in $U$ do not contain item set $Y$. Confidence of negative association rule, conf ( $X$ $Y$ ), can be calculated with $P\left(\begin{array}{ll}X & Y\end{array}\right) / P(X)$, where $P($.$) is the probability function. The support and confidence of itemsets$ are calculated during iterations. However, it is difficult to count the support and confidence of non-existing items in transactions. To avoid counting them directly, we can compute the measures through those of positive rules.

\section{RELATED WORK IN NEGATIVE ASSOCIATION RULE MINING}

We give a short description of the existing algorithms that can generate positive and negative association rules.

The concept of negative relationships mentioned for the first time in the literature by Brin et.al [11]. To verify the independence between two variables, they use the statistical test. To verify the positive or negative relationship, a correlation metric was used. Their model is chi-squared based. The chi-squared test rests on the normal approximation to the binomial distribution (more precisely, to the hyper geometric distribution). This approximation breaks down when the expected values are small.

A new idea to mine strong negative rules presented in [14]. They combine positive frequent itemsets with domain knowledge in the form of taxonomy to mine negative associations. However, their algorithm is hard to generalize since it is domain dependent and requires a predefined taxonomy. Finding negative itemsets involve following steps: (1) first find all the generalized large itemsets in the data (i.e., itemsets at all levels in the taxonomy whose support is greater than the user specified minimum support) (2) next identify the candidate negative itemsets based on the large itemsets and the taxonomy and assign them expected support. (3) in the last step, count the actual support for the candidate itemsets and retain only the negative itemsets .The interest measure RI of negative association rule $\mathrm{X} Y$, as follows $\mathrm{RI}=(\mathrm{E}[\operatorname{support}(\mathrm{X} \mathrm{U} \mathrm{Y})$ ]-support( $\mathrm{X}$ U Y $)$ )/support(X) Where $\mathrm{E}[\operatorname{support}(\mathrm{X})]$ is the expected support of an itemset $\mathrm{X}$.

A new measure called mininterest (the argument is that a

rule $A \quad B$ is of interest only if $\operatorname{supp}(A U B)$-supp $(A) \operatorname{supp}(B)$ mininterest $)$ added on top of the support-confidence framework[16]. They consider the itemsets (positive or negative) that exceed minimum support and minimum interest thresholds as itemsets of interest. Although, [8] introduces the "mininterest" parameter, the authors do not discuss how to set it and what would be the impact on the results when changing this parameter.

A novel approach has proposed in [15]. In this, mining both positive and negative association rules of interest can be decomposed into the following two sub problems, (1) generate the set of frequent itemsets of interest (PL) and the set of infrequent itemsets of interest (NL) (2) extract positive rules of the form A=>B in PL, and negative rules of the forms A B, A B and A B in NL. To generate PL, NL and negative association rules they developed three functions namely, fipi(), iipis() and CPIR().

The most common frame-work in the association rule generation is the "Support-Confidence" one. In [13], authors considered another frame-work called correlation analysis that adds to the support-confidence. In this paper, they combined the two phases (mining frequent itemsets and generating strong association rules) and generated the relevant rules while analyzing the correlations within each candidate itemset. This avoids evaluating item combinations redundantly. Indeed, for each generated candidate itemset, they computed all possible combinations of items to analyze their correlations. At the end, they keep only those rules generated from item combinations with strong correlation. If the correlation is positive, a positive rule is discovered. If the correlation is negative, two negative rules are discovered. The negative rules produced are of the form X $\quad \mathrm{Y}$ or $\mathrm{X} \quad \mathrm{Y}$ which the authors term as "confined negative association rules". Here the entire antecedent or consequent is either a conjunction of negated attributes or a conjunction of non-negated attributes.

An innovative approach has proposed in [12]. In this generating positive and negative association rules consists of four steps: (1) Generate all positive frequent itemsets $\mathrm{L}$ ( $\mathrm{P}_{1}$ ) (ii) for all itemsets I in $\mathrm{L}\left(\mathrm{P}_{1}\right.$ ), generate negative frequent itemsets of the form ( I1 I2 ) (iii) Generate all negative frequent itemsets I1 I2 (iv) Generate all negative frequent itemsets I1 I 2 and (v) Generate all valid positive and negative association rules. Authors generated negative rules without adding additional interesting measure(s) to support-confidence frame wor 


\section{International Journal of Innovative Research in Science, Engineering and Technology}

Vol. 1, Issue 1, November 2012

\section{ALGORITHM}

In this section we propose and explain our algorithm. Apriori algorithm has two steps, namely, the join step and the prune step. In join step all frequent itemsets of previous level joined itself to obtain candidate itemsets i.e., by joining $\mathrm{Lk}_{\mathrm{k}-1}$ to itself i.e.,

$$
C_{k}=L_{k-1} \quad L_{k}-1 \text {. }
$$

In pruning step, for each I $\mathrm{C}_{\mathrm{k}}$, it applies Apriori Property (an itemset is frequent if all its subsets are also frequent). Itemsets satisfying Apriori Property are called as valid itemsets and it is denoted by $\mathrm{PCK}_{\mathrm{K}}$. NCK can be obtained by replacing each literal in PCK by its corresponding negated item. For a valid candidate with $\mathrm{n}$ literals it produces $\mathrm{n}$ negative itemsets. Support of negative itemsets can be obtained from positive itemsets.

1) Algorithm: Negative Association Rules (NAR).

\section{INPUT:}

D:Transactional database, ms: minimum support, mc: minimum confidence

\section{OUTPUT: Positive and Negative Association Rules}

Method:

(1) $\quad$ L1=frequent-1-positive-itemsets(D) (2) $\quad \mathrm{N}_{1}=$ frequent-1-Negative-itemsets(D)

// complement frequent-1-positive-itemsets(D) (3) L=L1 U N1.

(6) // Generating $\mathrm{C}_{\mathrm{k}}$

(7) for each $11,12 \quad \mathrm{Lk}_{\mathrm{k}} 1$

(8) $\quad$ If $\left(11[1]=l_{2}[1]^{\wedge} \ldots \ldots \ldots . . .11[\mathrm{k}-2]=12[\mathrm{k}-2]^{\wedge} \mathrm{l} 1[\mathrm{k}-\right.$

\section{$1]<12[\mathrm{k}-1])$}

- Line 22-23, prune all items in $\mathrm{PC}_{\mathrm{k}}$ using support count and add to $\mathrm{Lk}_{\mathrm{k}}$, set of frequent $\mathrm{k}$-itemsets

- Line 24, find support count of all items in

$\mathrm{NC}_{\mathrm{k}}$ using $\mathrm{PC}_{\mathrm{k}}$ and $\mathrm{L}_{\mathrm{k}-1}$.

- $\operatorname{support}(A)=1-\operatorname{suuport}(A)$

- $\operatorname{support}(\mathrm{AU} \mathrm{B})=\operatorname{support}(\mathrm{A})-\operatorname{support}(\mathrm{AU} \mathrm{B})$

- $\operatorname{support}(\mathrm{AUB})=\operatorname{support}(\mathrm{B})-\operatorname{support}(\mathrm{AU} \mathrm{B})$

- $\operatorname{support}(\mathrm{AU} B) \quad$ B $=1-\operatorname{support}(\mathrm{A})-\operatorname{support}(\mathrm{B})+\operatorname{support}(\mathrm{A} U \mathrm{~B})$
(9)
$\mathrm{C}_{\mathrm{k}}=\mathrm{C}_{\mathrm{k}}$
$U\{\overline{11}[1]$
.....1 $[\mathrm{k}-2], 11[\mathrm{k}-1], 12[\mathrm{k}-1]\}$

is the set of all elements whose

(11) end for

(12) // Pruning using Apriori property (13)

for each (k-1)- subsets s of $\mathrm{c}$ in $\mathrm{C}_{\mathrm{k}}$ (14) If $\mathrm{s}$ is not a member of $\mathrm{L}_{\mathrm{k}-1}$

(15) $\mathrm{C}_{\mathrm{k}}=\mathrm{C}_{\mathrm{k}}-\{\mathrm{c}\}$

(16) end if

(17) end for

(18) $\quad \mathrm{PC}_{\mathrm{k}}=\mathrm{C}_{\mathrm{k}}$;

(19) for each $\mathrm{c}$ in $\mathrm{PCK}$

support minsupp.

- The generation of positive rules continues without disruption and the rich but valuable negative rules are produced as by-products of the Apriori process.

\section{EXPERIMENTAL RESULTS}

We tested proposed algorithm on a synthetic dataset to

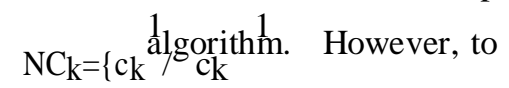

study the performance of the

illustrate the algorithm we hereunder provided the solution to

(21) //Pruning using Support Count

(22) Scan the database and find support for all $\mathrm{c}$ in $\mathrm{PC}_{\mathrm{K}}$

(23) $\mathrm{L}_{\mathrm{k}}=$ candidates in PCk that pass support threshold 


\section{International Journal of Innovative Research in Science, Engineering and Technology}

Vol. 1, Issue 1, November 2012

a typical example.

Example. Let us consider a small transactional table with 15 transactions and 9 items. In Table 1 a small transactional database is given $_{(24) \quad \text { Find support for all } \mathrm{ck}}$

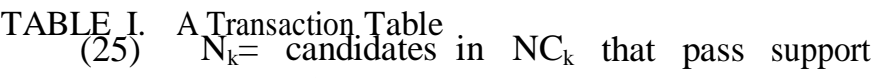
threshold

(26) $\mathrm{L}=\mathrm{L}_{\mathrm{k}} \mathrm{UN}_{\mathrm{k}}$

(27) \} 


\section{International Journal of Innovative Research in Science, Engineering and Technology}

Vol. 1, Issue 1, November 2012

-Line 1 generates positive-frequent-1-itemsets

- Line 2 generates negative-frequent-1-itemsets by complementing 1-itemsets obtained in Line 1

- $\quad$ Line 8 and 9 generates candidate itemsets $\mathrm{C}_{\mathrm{k}}$ using Apriori algorithm

- Line 13-15 pruning candidate itemsets in $\mathrm{C}_{\mathrm{K}}$ using Apriori property

- Lines 18, after pruning, the remaining elements are treated as valid candidates and is denoted by $\mathrm{PC}_{\mathrm{k}}$.

- Line 19-20, for each literal of this valid candidate, replace the literal with the corresponding negated literal, creates a new negative rule and denoted by $\mathrm{NC}_{\mathrm{k}}$. Each valid candidate with $\mathrm{n}$ number of literals in the antecedent will generate $\mathrm{n}$ new negative itemsets. For example a 3-itemset $\mathrm{ABC}$ will give 3 negative items $\mathrm{ABC}, \mathrm{A} \mathrm{BC}$, and $\mathrm{AB} \mathrm{C}$.

\begin{tabular}{|c|l|r|l|}
\hline L1 & SUPP & N1 & SUPP \\
\hline I2 & 0.4 & I2 & 0.6 \\
\hline I3 & 0.4 & I3 & 0.6 \\
\hline I4 & 0.27 & I4 & 0.73 \\
\hline I5 & 0.53 & I5 & 0.47 \\
\hline I6 & 0.33 & I6 & 0.67 \\
\hline I7 & 0.47 & I7 & 0.53 \\
\hline I8 & 0.27 & I8 & 0.73 \\
\hline
\end{tabular}

In the following tables, $\mathrm{LK}_{\mathrm{K}}$ denoted as positive frequent $\mathrm{k}$ - itemsets and $\mathrm{NK}_{\mathrm{K}}$ denoted as negative frequent k-itemsets. Consider minimum support as $20 \%$. In $\mathrm{N}_{\mathrm{K}}$ bold itemsets are infrequent negative itemsets.

Table II. Frequ Table III. Frequent 2-Positive Itemsets

\begin{tabular}{|c|c|c|c|c|c|c|c|}
\hline & I2 & I2 & I3 & I3 & I5 & I5 & I6 \\
L2 & I3 & I4 & I5 & I7 & I6 & I7 & I7 \\
\hline SUPP & 0.2 & 0.2 & 0.2 & 0.2 & 0.2 & 0.33 & 0.2 \\
\hline
\end{tabular}

Table IV: Frequent 2-Negative Itemsets

\begin{tabular}{|c|c|cc|c|}
\hline N2 & SUPP & \multicolumn{2}{|c|}{ N2 } & SUPP \\
\hline I2 I3 & 0.2 & I2 & I3 & 0.2 \\
\hline I2 I4 & $\mathbf{0 . 0 7}$ & I2 & I4 & 0.2 \\
\hline I3 I5 & 0.33 & I3 & I5 & 0.2 \\
\hline I3 I7 & 0.27 & I3 & I7 & 0.2 \\
\hline I5 I6 & $\mathbf{0 . 1 3}$ & I5 & I6 & 0.33 \\
\hline I5 I7 & $\mathbf{0 . 1 4}$ & I5 & I7 & 0.2 \\
\hline I6 I7 & 0.27 & I6 & I7 & $\mathbf{0 . 1 3}$ \\
\hline
\end{tabular}

Table V: Frequent 3-Positive Itemsets

\begin{tabular}{|c|c|}
\hline L3 & SUPP \\
\hline I3I5I7 & 0.2 \\
\hline
\end{tabular}

Table VI: Frequent 3-Negative Itemsets

\begin{tabular}{|c|c|}
\hline N3 & SUPP \\
\hline I3I5I7 & $\mathbf{0 . 1 3}$ \\
\hline I3 I5I7 & $\mathbf{0}$ \\
\hline I3I5 I7 & $\mathbf{0}$ \\
\hline
\end{tabular}




\section{International Journal of Innovative Research in Science, Engineering and Technology \\ Vol. 1, Issue 1, November 2012}

\section{CONCLUSION AND FUTURE WORK}

In this paper, we proposed an algorithm that mines both positive and negative association rules. Our method generates positive and negative rules with existing support-confidence framework and no extra scan is required for mining negative association rules. We conducted experiments on synthetic data set. It is producing larger number of negative rules. In future we wish to conduct experiments on real datasets and compare the performance of our algorithm with other related algorithms.

\section{REFERENCES}

[1] R. Agrawal and R. Srikant. Fast algorithms for mining associationrules. In VLDB, Chile, September 1994.

[2] J. Han, J. Pei, and Y. Yin. Mining frequent patterns without candidate generation. In SIGMOD, dallas, Texas, 2000.

[3] C. Blakeand C. Merz. UCI repository of machine learning databases.

[4] S.Brin, R. Motwani, and C.Silverstein. Beyond market baskets: Generalizing association rules to correlations. In ACM SIGMOD, Tucson, Arizona, 1997.

[5] D. Thiruvady and G. Webb. Mining negative association rules using grd. In PAKDD, Sydney, Australia, 2004

[6] B.Ramasubbareddy, A.Govardhan, and A.Ramamohanreddy. Adaptive approaches in mining negative association rules. In intl. conference on ITFRWP-09, India Dec-2009.

[7] Goethals, B., Zaki, M., eds.: FIMI'03: Workshop on Frequent Itemset Mining Implementations. Volume 90 of CEUR Workshop Proceedings series. (2003) http://CEUR-WS.org/Vol-90/.

[8] Teng, W., Hsieh, M., Chen, M.: On the mining of substitution rules for statistically dependent items. In: Proc. of ICDM. (2002) 442-449

[9] Tan, P., Kumar, V.: Interestingness measures for association patterns: A perspective.In: Proc. of Workshop on Postprocessing in Machine Learning and Data Mining. (2000)

[10] Gourab Kundu, Md. Monirul Islam, Sirajum Munir, Md. Faizul Bari ACN: An Associative Classifier with Negative Rules 11th IEEE International Conference on Computational Science and Engineering, 2008.

[11] Brin,S., Motwani,R. and Silverstein,C., “ Beyond Market Baskets: Generalizing Association Rules to Correlations," Proc. ACM SIGMOD Conf., pp.265-276, May 1997.

[12] Chris Cornelis, peng Yan, Xing Zhang, Guoqing Chen: Mining Positive and Negative Association Rules from Large Databases, IEEE conference 2006.

[13] M.L. Antonie and O.R. Za" ane, "Mining Positive and Negative Association Rules: an Approach for Confined Rules", Proc. Intl. Conf. on Principles and Practice of Knowledge Discovery in Databases, 2004, pp 27-38.

[14] Savasere, A., Omiecinski,E., Navathe, S.: Mining for Strong negative associations in a large data base of customer transactions. In: Proc. of ICDE. (1998) 494502.

[15] Wu, X., Zhang, C., Zhang, S.: efficient mining both positive and negative association rules. ACM Transactions on Information Systems, Vol. 22, No.3, July 2004,Pages 381-405.

[16] Wu, X., Zhang, C., Zhang, S.: Mining both positive and negative association rules.In: Proc. of ICML. (2002) 658-665

[17] Yuan,X., Buckles, B.,Yuan, Z.,Zhang, J.:Mining Negative Association Rules. In: Proc. of ISCC. (2002) 623-629.

[18] Honglei Zhu, Zhigang Xu: An Effective Algorithm for Mining Positive and Negative Association Rules. International Conference on Computer Science and Software Engineering 2008.

[19] Pradip Kumar Bala:A Technique for Mining Negative Association Rules Proceedings of the 2nd Bangalore Annual Compute Conference (2009).

[20] Data Mining: Concepts and Techniques Jiawei Han, Micheline Kamberent 1-Positive And Negative Itemsets 